\title{
Planetary Boundaries: Separating Fact from Fiction. A response to Montoya et al.
}

\author{
Johan Rockström ${ }^{1 *}$, Katherine Richardson ${ }^{2}$, Will Steffen ${ }^{3}$, Georgina Mace ${ }^{4}$
}

\author{
${ }^{1}$ Stockholm Resilience Centre, Stockholm University \\ ${ }^{2}$ Center for Macroecology, Evolution and Climate, University of Copenhagen, Denmark \\ 3 the Australian National University, ANU, Australia \\ ${ }^{4}$ Centre for Biodiversity \& Environment Research (CBER), University College London, UK \\ *corresponding author, johan.rockstrom@su.se
}

\begin{abstract}
A recent article by Montoya et al. [1] in TREE presents a vitriolic and highly opinionated critique of the planetary boundaries (PB) framework based on a fundamental misrepresentation of the framework and a repetition of earlier ill-informed and misguided attacks on it. Herein we set the record straight and note more positive ways forward.
\end{abstract}

Planetary boundaries and tipping points. Montoya et al. [1] conflate PBs with tipping points. Furthermore, they mistakenly claim that the PB framework relies on the assumption of thresholds or tipping points. They state: "One solution (to environmental research and policy challenges) embraces the notion of Planetary Boundaries arguing that global environmental processes very generally have 'tipping points'. These are catastrophes involving thresholds beyond which there will be rapid transitions to new states that are very much less favorable to human existence than current states."

The fact is that both major PB papers [2,3] state clearly that a PB is not a global threshold or tipping point: "A planetary boundary as originally defined is not equivalent to a global threshold or tipping point [3]."

In addition, throughout their paper Montoya et al. [1] focus on knocking down the 'straw man' of tipping points in relation to the biodiversity (biosphere integrity) PB. In particular, they claim that: "The rate of human-caused extinctions - now 100-1000-fold the natural background rate - is one of two of the nine global processes deemed to have exceeded a purported tipping point of 10-fold background."

The fact is that neither PB paper [2,3] has ever claimed that there is a global-level tipping point for biodiversity. In fact, we have gone out of our way to emphasize that we do not have scientifically established evidence of such tipping point [3]: "Not all Earth-system processes included in the PB approach have singular thresholds at the global/continental/ocean basin level.... Examples of such processes are land-system change, freshwater use, changes in biosphere integrity (rate of biodiversity loss in [2]), and changes in other biogeochemical flows in addition to carbon (e.g., nitrogen and phosphorus)." 
It is absolutely clear that the Montoya et al. [1] 'definition' of the PB framework is blatantly incorrect. Indeed, one can only conclude that they have either not read or deliberately misrepresented the PB framework as described in [2,3]. Either explanation is unacceptable for a scholarly critique in the peer-reviewed literature, and renders their critique both meaningless and not applicable to the PB framework as it actually is formulated. Nevertheless, one further misrepresentation and one additional point are useful to consider.

Purpose of planetary boundaries. The PB framework is designed explicitly for the global level only (as the name clearly indicates). It is a scientific approach for (i) identifying the processes that regulate the state of the Earth System, and (ii) proposing boundaries for these processes to maintain a Holocene-like (interglacial) state of the Earth System [2,3]. The PB framework is complementary to the myriad methods and policies for ecosystem management at sub-global levels, and is not meant to either replace or override these necessary and important approaches, as erroneously inferred throughout [1]. There is no doubt, however, that attempts have been made - and continue to be made - to apply the PB framework to levels lower than, and sometimes much lower than, the global.

The fact is, however, that such 'downscaling' has never been either proposed or encouraged in the PB framework papers [2,3]. By necessity, six of the PBs indeed have a two-level set of control variables and boundaries, with large biome/ocean basin levels in addition to the global level [3]. This sub-global level recognizes that to understand the functioning and stability of a complex system such as the Earth System, the influence of processes, particularly feedback processes, at levels below that of the system itself can play an important role in influencing or regulating the system's functioning [4]. We were certainly aware of potential misunderstanding of this point, and so included an explicit caution in [3]: "We emphasize that our sub-global level focus is based on the necessity to consider this level to understand the functioning of the Earth System as a whole. The PB framework is therefore meant to complement, not replace or supersede, efforts to address local and regional environmental issues."

Again, Montoya et al. [1] have apparently either not read or chosen to deliberately mispresent what the PB framework actually says [3].

Biodiversity and ecosystem/Earth System functioning. Montoya et al. [1] appear to be somewhat confused in taking both a linear cause-effect approach to ecosystems (e.g. "...which species are vital to which processes") and systems approaches (e.g., "resilience (how fast systems recover), resistance (how much they change), variability (how much they fluctuate over time), and persistence (how long they persist)"). While both framings have their place, it is the complex systems approach that is central to the PB framework $[2,3]$. Here we agree completely with Montoya et al. [1]: "...mounting evidence demonstrates the patterns and mechanisms by which biodiversity loss alters the provision of functions and the stability of ecosystems."

In precisely the same way, the PB framework is concerned with the role of the biosphere (consisting of myriad ecosystems) in regulating the stability of the Earth System. In fact, for 
most of the 4.5 billion-year history of the Earth System, the geosphere and biosphere have co-evolved as a single, interacting system, emphasizing the very important role that the biosphere plays in the functioning and stability of the Earth System as a whole [5].

Finally, as for more positive ways forward, we have always welcomed constructive criticism of the PB framework, and indeed this has often led to fruitful collaboration to improve the framework as the underpinning science advances. Examples include improvements in both the phosphorus [6] and nitrogen [7] boundaries. The updated biosphere integrity boundary [3] has also benefitted significantly from constructive criticism from, and follow-up collaboration with, the biodiversity research community itself [8]. Curiously, this important paper for the biosphere integrity PB was not cited in [1], and there is no evidence that any of the insights from [8] have even been considered in the Montoya et al. critique [1].

Given the very constructive collaboration we've had, and continue to have, with many scientific communities interested in the PB framework, it is regrettable that Montoya et al. [1] have, instead, taken such a personal, confrontational, and subjective approach in their paper. Particularly as the planetary boundaries framework continues to be science in progress, as recognised by Steffen et al. [3], where we see it as a top priority to continue working with the ecological modelling and biodiversity science community to further advance our understanding of the role of biodiversity for Earth system stability.

\section{References}

1. Montoya, J et al. (2017) Planetary Boundaries for biodiversity: Implausible science, pernicious policies. Trends in Ecology and Evolution (TREE) xx, xxx-xxx

2. Rockström, J et al. (2009) A safe operating space for humanity. Nature 461, 472-475

3. Steffen, W et al. (2015) Planetary Boundaries: Guiding human development on a changing planet. Science 347, DOI: 10.1126/science.1259855

4. Scheffer, M (2009) Critical Transitions in Nature and Society. Princeton University Press, $384 \mathrm{pp}$.

5. Lenton, TM (2015) Earth System Science. A Very Short Introduction. Oxford, Oxford University Press, 153 pp.

6. Carpenter, $S$ and Bennett, EM (2011) Reconsideration of the planetary boundary for phosphorus. Environ. Res. Lett. 6, doi:10.1088/1748-9326/6/1/014009

7. de Vries, W et al. (2013) Assessing planetary and regional nitrogen boundaries related to food security and adverse environmental impacts. Curr. Opinion Environ. Sust. 5, 392-402

8. Mace, GM et al. (2014) Approaches to defining a planetary boundary for biodiversity. Global Environmental Change 28, 289-297. DOI: 10.1016/j.gloenvcha.2014.07.009 
